\title{
Collisional dissipation of Alfvén waves in a partially ionised solar chromosphere
}

\author{
J. E. Leake ${ }^{1}$, T. D. Arber ${ }^{1}$, and M. L. Khodachenko ${ }^{2}$ \\ 1 Space and Astrophysics group, Dept of Physics, University of Warwick, Gibbet Hill Road, CV4 7AL, UK \\ e-mail: J.E.Leake@warwick . ac . uk \\ 2 Space Research Institute, Austrian academy of sciences, 8042 Graz, Austria \\ e-mail: maxim.khodachenko@oeaw.ac.at
}

Received 13 May 2005 / Accepted 19 July 2005

\section{ABSTRACT}

Certain regions of the solar atmosphere are at sufficiently low temperatures to be only partially ionised. The lower chromosphere contains neutral atoms, the existence of which greatly increases the efficiency of the damping of waves due to collisional friction momentum transfer. More specifically the Cowling conductivity can be up to 12 orders of magnitude smaller than the Spitzer value, so that the main damping mechanism in this region is due to the collisions between neutrals and positive ions (Khodachenko et al. 2004, A\&A, 422, 1073). Using values for the gas density and temperature as functions of height taken from the VAL C model of the quiet Sun (Vernazza et al. 1981, ApJS, 45, 635), an estimate is made for the dependance of the Cowling conductivity on height and strength of magnetic field. Using both analytic and numerical approaches the passage of Alfvén waves over a wide spectrum through this partially ionised region is investigated. Estimates of the efficiency of this region in the damping of Alfvén waves are made and compared for both approaches. We find that Alfvén waves with frequencies above $0.6 \mathrm{~Hz}$ are completely damped and frequencies below $0.01 \mathrm{~Hz}$ unaffected.

Key words. Sun: chromosphere - magnetic fields - stars: atmospheres - plasmas - waves

\section{Introduction}

MHD waves are vital phenomena in the study of the solar atmosphere. They have been suggested as a source of heating of both the chromosphere and the corona (Goodman 2000; Piddington 1956), based on the damping of wave energy due to dissipation effects.

Three main energy dissipation mechanisms in the chromosphere have been studied in previous work, namely collisional effects, viscous effects and thermal conductivity effects. Viscous damping of MHD waves is caused by the momentum transfer from the thermal motion of particles. Collisional friction is caused by the relative velocities of the species in the plasma. While thermal conductivity is also related to kinetic effects and momentum transfer. Khodachenko et al. (2004) and Khodachenko et al. (2005) showed that in the partially ionised plasma of the lower solar chromosphere, collisional effects are far more important than the other two. Also the presence of neutrals means that of all the collisions to be considered it is the collisions between neutrals and ions that are the most important component of this mechanism.

Alfvén waves are a specific type of MHD wave. Previous work on the generation and propagation of Alfvén waves has included the simulation of high frequency waves generated by photospheric motions and the damping of these waves in the partially ionised chromosphere above. This damping was suggested as an explanation of the formation of spicules (DePontieu \& Haerendel 1998; DePontieu 1999; James et al. 2003). Spicules are high, thin jet like structures consisting of chromospheric plasma. They can reach heights of between 5000 and $9500 \mathrm{~km}$ whereupon they either fall back or fade in the hot corona. This work involved the numerical simulation of Alfvén waves of frequencies around $0.5 \mathrm{~Hz}$ damped by ion-neutral collisions in the WKB approximation. This approximation assumes that over a single wavelength there is no spatial variation of variables. More specifically, the wavelength itself does not change much on its own scale and the damping scale is much larger than the wavelength. De Pontieu et al. (2001) analytically calculated the damping time for Alfvén waves of varying frequencies in model chromospheres of various solar structures, given estimates for the plasma parameters in these regions. They estimated that frequencies above about $0.1 \mathrm{~Hz}$ are unable to penetrate through to the corona from the photosphere.

Other types of MHD waves in the solar atmosphere have also been studied. Linear analysis of slow MHD waves in the frequency range below $3.5 \times 10^{-3} \mathrm{~Hz}$ has been used to show the damping as a possible heating mechanism for the chromospheric network. The dissipation of the currents asscoiated with these waves, which is suggested to occur in thin magnetic flux 
tubes with strengths of $700 \mathrm{G}$ to $1500 \mathrm{G}$, heats the tubes and hence the chromospheric network (Goodman 2000).

The passage of waves through both the chromosphere and corona has been studied using measurements of magnetic bright point (MBP) positions in the photosphere. A frequency power spectrum for horizontal motions (Cranmer \& Ballegooijen 2005) at the photospheric level were derived in the frequency range $10^{-5}$ to $0.1 \mathrm{~Hz}$. Using these as a lower boundary condition, a WKB approximation was applied to derive power spectra at different heights in the atmosphere to show the effective damping of waves in different regions. It was shown that waves in this range $\left(10^{-5}\right.$ to $\left.0.1 \mathrm{~Hz}\right)$ may be evident up to a few solar radii which suggests that horizontal perturbations in this frequency range may be unaffected by the partially ionised chromosphere.

Higher frequency waves have also been detected in the solar atmosphere. Waves of frequencies up to $0.1 \mathrm{~Hz}$ have been detected in the upper chromosphere (Deforest 2004) and transition region. These waves were visible in the TRACE $1600 \AA$ passband. The exact source of these waves is uncertain and they are not energetically significant to the chromosphere. High frequency waves $(0.1 \mathrm{~Hz})$ in the lower chromosphere have proved harder to find (Fossum \& Carlsson 2005). It has been suggested that high frequency Alfvén waves in the range 1 to $800 \mathrm{~Hz}$ can be created by small scale reconnection in the chromospheric network, and their dissipation was considered as a possible heating mechanism for the corona above (Marsch \& Tu 1997).

Here we present both analytical estimates and full MHD simulations of the efficiency of damping of Alfvén waves in the partially ionised chromosphere. We give estimates of the effective filter function for the damping of Alfvén waves. This damping is assumed to be due to the collisions of neutrals and positive ions in a model hydrogen plasma. The Alfvénic disturbances are assumed to be created at the photospheric level by horizontal motions on the surface.

Given values for the plasma density and temperature in the lower chromosphere taken from the VAL C model of the quiet Sun (Vernazza et al. 1981), the Cowling conductivity is estimated. Using the linear damping approximation (Braginskii 1965) the damping decrement for upwardly travelling waves is calculated and the efficiency of this region in damping the waves is estimated.

Then full MHD numerical simulations of linear Alfvén waves in a model atmosphere which contains a partially ionised chromosphere are performed. We investigate the efficiency of collisional damping for a range of frequency of wave. The numerical results are compared with the theoretical estimates and are shown to be in agreement with the linear damping approximation for low frequency waves but differ for higher frequency waves.

\section{Collisional friction damping in a partially ionised plasma}

To incorporate the damping of MHD waves due to ionneutral collisions in a partially ionised plasma, the generalized
Ohm's law should be included in the governing equations. (Cowling 1957; Braginskii 1965).

$$
\begin{aligned}
\boldsymbol{E}+(\boldsymbol{v} \wedge \boldsymbol{B})= & \frac{1}{\sigma} \frac{\nabla \wedge \boldsymbol{B}}{\mu_{0}} \\
& -\frac{\xi^{2}}{\alpha_{\mathrm{n}}}\left[\frac{\nabla \wedge \boldsymbol{B} \wedge \boldsymbol{B} \wedge \boldsymbol{B}}{\mu_{0}}\right] \\
= & \frac{1}{\sigma} \boldsymbol{j}+\frac{\xi^{2} B_{0}^{2}}{\alpha_{\mathrm{n}}} \boldsymbol{j}_{\perp}
\end{aligned}
$$

where $\boldsymbol{v}$ is the fluid velocity, $\boldsymbol{B}$ is the magnetic field, $\boldsymbol{E}$ is the electric field, $\boldsymbol{j}=(\nabla \wedge \boldsymbol{B}) / \mu_{0}$ is the current density and $\boldsymbol{j}_{\perp}$ is the component perpendicular to the magnetic field.

The conductivity is defined by

$$
\sigma=\frac{n_{\mathrm{e}} e^{2}}{m_{\mathrm{e}}\left(v_{\mathrm{ei}}^{\prime}+v_{\mathrm{en}}^{\prime}\right)}
$$

and

$\alpha_{\mathrm{n}}=m_{\mathrm{e}} n_{\mathrm{e}} v_{\text {en }}^{\prime}+m_{\mathrm{i}} n_{\mathrm{i}} v_{\text {in }}^{\prime}$.

The number densities of species (ion, electron, neutral) are given by $n_{\mathrm{i}}, n_{\mathrm{e}}, n_{\mathrm{n}}$ respectively, and the masses by $m_{\mathrm{i}}, m_{\mathrm{e}}, m_{\mathrm{n}}$.

$\xi_{\mathrm{n}}=\frac{m_{\mathrm{n}} n_{\mathrm{n}}}{m_{\mathrm{n}} n_{\mathrm{n}}+m_{\mathrm{i}} n_{\mathrm{i}}}$

is the relative density of neutrals, and $v_{\text {ie }}^{\prime}, v^{\prime}{ }_{\text {ie }}$ and $v_{\text {ie }}^{\prime}$ are the effective collisional frequencies defined by

$v_{k l}^{\prime}=\frac{m_{l}}{m_{l}+m_{k}} v_{k l}$

where $k=\mathrm{e}, \mathrm{i}, l=\mathrm{i}, \mathrm{n}$. Following the example of Spitzer (1962), the collisional frequencies of ions and electrons with neutrals are estimated by

$$
\begin{aligned}
& v_{\mathrm{in}}=n_{\mathrm{n}} \sqrt{\frac{8 K_{\mathrm{B}} T}{\pi m_{\mathrm{in}}}} \Sigma_{\mathrm{in}} \\
& v_{\mathrm{en}}=n_{\mathrm{n}} \sqrt{\frac{8 K_{\mathrm{B}} T}{\pi m_{\mathrm{en}}}} \Sigma_{\mathrm{en}}
\end{aligned}
$$

where

$m_{\mathrm{in}}=\frac{m_{\mathrm{i}} m_{\mathrm{n}}}{m_{\mathrm{i}}+m_{\mathrm{n}}}$.

and $K_{\mathrm{B}}$ is Boltzmann's constant. The collisional frequency of electrons and ions is given by

$v_{\mathrm{ei}}=5.89 \times 10^{-24} \frac{n_{\mathrm{i}} \Lambda Z^{2}}{T^{3 / 2}}$

(Spitzer 1962). $\Sigma_{\text {in }}=5 \times 10^{-19} \mathrm{~m}^{2}, \Sigma_{\text {en }}=10^{-19} \mathrm{~m}^{2}$ are the ionneutral and electron-neutral cross-sections respectively. Here $\Lambda$ is the Coulomb logarithm.

In the generalised Ohm's law (Eqs. (1) and (2)) we have neglected the pressure term, as the chromospheric plasma is relatively cold, and the Hall term. The Hall term can be dropped from the generalised Ohm's law if the plasma is magnetised, i.e. if the ions and electrons are tightly bound to the magnetic field. In terms of the ion-gyrofrequency and the collision time this condition can be written as

$\omega_{\mathrm{i}} \tau \gg 1$. 


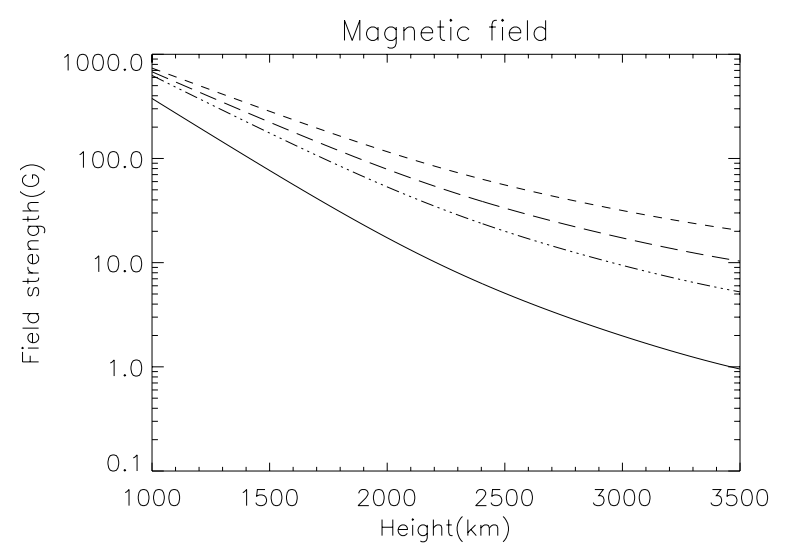

Fig. 1. The variation of the model background magnetic field with height. The four curves relate to the value of the exponent $\alpha=0.3$ (small dash line), 0.35 (big dash line), 0.4 (dot-dash line) and 0.6 (solid line).

Using the definition of the ion-neutral collision frequency this is equivilant to

$\frac{e B}{m_{\mathrm{i}}} \sqrt{\frac{\pi m_{\mathrm{i}}}{16 k_{\mathrm{B}} T}} \frac{1}{n_{\mathrm{n}} \Sigma_{\mathrm{in}}} \gg 1$

which depends on the strength of the magnetic field as well as the temperature and number density. Using values for temperature and number density taken from the VALC model of the Sun (Vernazza et al. 1981), and various models of magnetic field strengths in the chromosphere (see Fig. 1), this condition is satisfied everywhere in the chromosphere. Khodachenko \& Zaitsev (2001) showed that this condition does not hold in the upper photosphere where the plasma is more weakly ionised. In Sect. 3 we will show that the damping mechanism we are considering is not effective in the photosphere, and thus we only study Alfvén waves in the chromosphere for which the neglect of the Hall term is valid.

If the plasma is assumed to be entirely composed of hydrogen then the expressions can be simplified by taking $m_{\text {in }}=$ $m_{\mathrm{n}} / 2, \xi_{\mathrm{n}}=\rho_{\mathrm{n}} / \rho$ resulting in

$\alpha_{\mathrm{n}}=\frac{1}{2} \xi_{\mathrm{n}}\left(1-\xi_{\mathrm{n}}\right) \frac{\rho^{2}}{m_{\mathrm{n}}} \sqrt{\frac{16 k_{\mathrm{B}} T}{\pi m_{\mathrm{i}}}} \Sigma_{\text {in }}$.

Using the definition for the Cowling conductivity as

$\sigma_{\mathrm{c}}=\frac{\sigma}{1+\frac{\xi_{\mathrm{n}}^{2} B_{0}^{2} \sigma}{\alpha_{\mathrm{n}}}}$

and defining the Coulomb and Cowling resistivity as $\eta=1 / \sigma$ and $\eta_{\mathrm{c}}=1 / \sigma_{\mathrm{c}}$ respectively it is trivial to show that

$\frac{\xi_{\mathrm{n}}^{2} B_{0}^{2}}{\alpha_{\mathrm{n}}}=\eta_{\mathrm{c}}-\eta$.

Thus Eq. (2) can be be written as

$$
\begin{aligned}
\boldsymbol{E}+(\boldsymbol{v} \wedge \boldsymbol{B}) & =\eta \boldsymbol{j}+\left(\eta_{\mathrm{c}}-\eta\right) \boldsymbol{j}_{\perp} \\
& =\eta \boldsymbol{j}_{\|}+\eta_{\mathrm{c}} \boldsymbol{j}_{\perp}
\end{aligned}
$$

and the frictional Joule heating term for this plasma (Braginskii 1965) is then given by

$$
\begin{aligned}
Q & =(\boldsymbol{E}+(\boldsymbol{v} \wedge \boldsymbol{B})) \cdot \boldsymbol{j} \\
& =\eta j_{\|}{ }^{2}+\eta_{\mathrm{c}} j_{\perp}{ }^{2}
\end{aligned}
$$

with $j_{\perp}$ and $j_{\|}$being the components of $\boldsymbol{j}$ perpendicular and parallel to $\boldsymbol{B}$. Thus in a partially ionised plasma the Coulomb resistivity acts to diffuse currents parallel to the magnetic field and the Cowling resistivity acts to diffuse currents perpendicular to the magnetic field. The damping decrement $(\delta)$ for this effect was derived by Braginskii (1965) for Alfvén waves propagating with angular frequency $\omega$ and wavenumber $\boldsymbol{k}$ and is given by

$\frac{1}{\tau}=2 \omega \delta_{\text {Joule }}=\frac{1}{\mu_{0}} \eta k_{\perp}^{2}+\frac{1}{\mu_{0}} \eta_{\mathrm{c}} k_{\|}^{2}$

Khodachenko et al. (2004, 2005) showed that for typical magnetic field strengths and using the VAL C model for the plasma parameters, the ratio of Coulomb to Cowling resistivity can vary from 1 at the solar surface $(z=0)$ down to $10^{-12}$ at heights of $2000 \mathrm{~km}$. For the partially ionised chromosphere

$\frac{\eta}{\eta_{\mathrm{c}}}=\frac{1}{1+\xi_{\mathrm{n}}^{2} \frac{B_{0}^{2}}{\eta \alpha_{\mathrm{n}}}} \ll 1$.

Hence when considering frictional damping mechanisms, the ion-neutral collisions are by far the most important.

\section{Analytic approach}

In order to evaluate the expression for the Cowling conductivity $\eta_{\mathrm{c}}$ an estimate for the neutral fraction $\xi_{\mathrm{n}}$ is required (see Eq. (15)). Although the VAL C model gives us the ionisation degree for the Sun our model is a hydrogen plasma. Thus $\xi_{\mathrm{n}}$ is calculated from the density and temperature values only of the VAL C model. Here we follow the method of De Pontieu (1999), assuming a hydrogen plasma where the number of electrons is equal to the number of protons $\left(n_{\mathrm{i}}=n_{\mathrm{e}}\right)$.

The solar chromosphere, and spicules especially are not in LTE. Hence a simple one-level model for the hydrogen atom is inadequate for these conditions (Pottasch \& Thomas 1959). A two-level model is used instead for the hydrogen atom, so that the ionisation equation (Brown 1973) is

$n \frac{\partial x}{\partial t}=\sum\left(C_{j}+P_{j}+C_{j}^{*}+P_{j}^{*}\right)$

where $n$ is local number density, and $x$ is the ionisation degree. $C_{j}$ and $P_{j}$ are the ionisation rates from level $j$ due to thermal collisions and local radiation field respectively. $C_{j}{ }^{*}$ and $P_{j}{ }^{*}$ are the ionisation rates by non-thermal particles and external radiation field. In the first approximation photoionisation from level 2 to level 1 is provided by the external field alone, so that $P_{1}=P_{1}{ }^{*}=0$. This external field is simply the photospheric radiation field at temperature $T_{\mathrm{R}}$, suitably diluted by factor $w$. The ionisation equation can be further simplified by noting that thermal collision ionisation is unimportant when compared to photoionisation (Ambartsuyan 1958). Thus the ionisation equation is balanced by photo-ionisation from 
level 2 to level 1 and spontaneous recombination for the return route.

$n \frac{\partial x}{\partial t}=P_{2_{+}}^{*}-P_{2-}$.

The steady state solution to this equation is given by (Thomas \& Athay 1961)

$$
\begin{aligned}
\frac{n_{\mathrm{i}}^{2}}{n_{\mathrm{n}}} & =\frac{f(T)}{b(T)} \\
f(T) & =\frac{\left(2 \pi m_{\mathrm{e}} K_{\mathrm{B}} T\right)^{\frac{3}{2}}}{h^{3}} \exp \left(-\frac{X_{i}}{K_{\mathrm{B}} T}\right) \\
b(T) & =\frac{T}{w T_{\mathrm{R}}} \exp \left[\frac{X_{i}}{4 K_{\mathrm{B}} T}\left(\frac{T}{T_{\mathrm{R}}}-1\right)\right]
\end{aligned}
$$

where $T_{\mathrm{R}}$ is the temperature of the photospheric radiation field and $w=0.5$ is its dilution factor.

Using the solar atmospheric plasma variables given by the VAL C model the neutral fraction $\xi_{\mathrm{n}}$ and the profile of $\eta_{\mathrm{c}}$ with height can be calculated for a given magnetic field profile.

As we intend to simulate the motion of waves in the chromospheric region of the solar atmosphere, we choose as our magnetic field model a spreading vertical flux tube representing an open magnetic structure with its footpoint in the photosphere. As Alfvén waves propagate along a fieldline we can restrict ourselves to one spatial dimension and need only define the variation of the magnetic field as a function of distance along the fieldline. Thus the horizontal expansion of the tube does not enter explicitly into our calculations. We choose a magnetic field profile to reflect the conservation of flux as the tube expands. A power law dependance on density is chosen

$B=B_{0}\left(\frac{\rho}{\rho_{\mathrm{ph}}}\right)^{\alpha}$

where $\rho_{\mathrm{ph}}$ is the photospheric density $2.7 \times 10^{-4} \mathrm{~kg} / \mathrm{m}^{3}$ and $B_{0}$ is $1200 \mathrm{G}$. This profile is chosen so that at the base of the domain the magnetic field is approximately $1000 \mathrm{G}$ and falls to a value of about $10 \mathrm{G}$ at a height of $3000 \mathrm{~km}$. This is a simplistic model for the magnetic field but captures the decrease with height and enables comparison of analytic and numerical results. Martinez-Pillet et al. (1997) showed that the field strength in flux tubes can drop to as little as $300 \mathrm{G}$ at heights of only $300 \mathrm{~km}$ so we use this as the minimum value of magnetic field at this height for our models. The magnetic field profile for four different power law profiles are shown in Fig. 1. A typical calulated value of $\eta_{\mathrm{c}}$ as a function of height for the case when $\alpha=0.3$ is shown in Fig. 2. Khodachenko et al. (2005) calculated $\frac{\eta}{\eta_{\mathrm{c}}}$ for the solar atmosphere given constant magnetic fields. The ratio at a height of $1500 \mathrm{~km}$ can be as low as $10^{-6}$ giving a value of the Coulomb resistivity of $8 \times 10^{-5} \mathrm{~m}^{2} / \mathrm{s}$.

Equation (18) gives the inverse damping timescale of Alfvén waves with angular frequency $\omega$ and wavenumber $k_{\|}$ along the magnetic field, propagating through a region of constant $\eta_{\mathrm{c}}$.

$\frac{1}{\tau}=2 \omega \delta=\frac{\eta_{\mathrm{c}}}{\mu_{0}} k_{\|}^{2}$

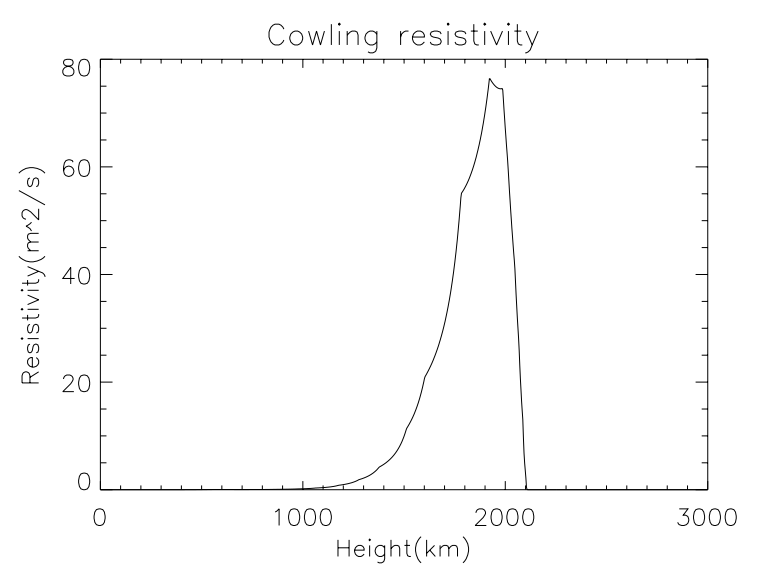

Fig. 2. Cowling resistivity as a function of height calculated from VAL $C$ model of the quiet Sun and varying $B_{0}$ with height according to Eq. (25) with $\alpha=0.3$.

The linear MHD analysis used to obtain damping decrements (Braginskii 1965) assumes that the damping decrement $\delta$ is much less than 1 . This implies that

$\delta=\frac{\eta_{\mathrm{c}} k_{\|}^{2}}{2 \mu_{0} \omega} \ll 1$

or that

$\omega \ll \omega_{\text {crit }}=\frac{2 \mu_{0} c_{\mathrm{A}}^{2}}{\eta_{\mathrm{c}}}$.

For the model used here both $\eta_{\mathrm{c}}$ and the Alfvén speed

$c_{A}=\frac{B}{\left(\mu_{0} \rho\right)^{\frac{1}{2}}}$

are functions of height so the damping decrement also varies with height. Over a small timestep $\mathrm{d} t$ the amplitude of the propagating wave changes by

$$
\begin{aligned}
A & =A_{0} \exp \left(-\frac{\mathrm{d} t}{\tau}\right) \\
& =A_{0} \exp \left(-\mathrm{d} t \frac{\eta_{\mathrm{c}}(z)}{\mu_{0}} k_{\|}(z)^{2}\right) \\
& =A_{0} \exp \left(-\mathrm{d} z \frac{\eta_{\mathrm{c}}(z)}{\mu_{0} c_{\mathrm{A}}(z)^{3}} \omega^{2}\right) .
\end{aligned}
$$

By integrating over the partially ionised region where $\eta_{\mathrm{c}}$ is nonzero (Fig. 2) we get an estimate for the total change in amplitude due to the damping mechanism

$E=\frac{A_{0}-A}{A_{0}}=1-\exp \left[-\omega^{2} \int \frac{\eta_{\mathrm{c}}(z)}{\mu_{0} c_{\mathrm{A}}(z)^{3}} \mathrm{~d} z\right]$

where the integral can be performed numerically. The results from this approximation are shown in Fig. 3 for the four different magnetic field models (different $\alpha$ ). From these estimates it appears that in the absence of any other damping mechanisms and stratification effects, high frequency Alfvén waves are unable to pass through this partially ionised region without being completely damped. For the magnetic field model with $\alpha=0.3$ this can be as low as $0.1 \mathrm{~Hz}$. 


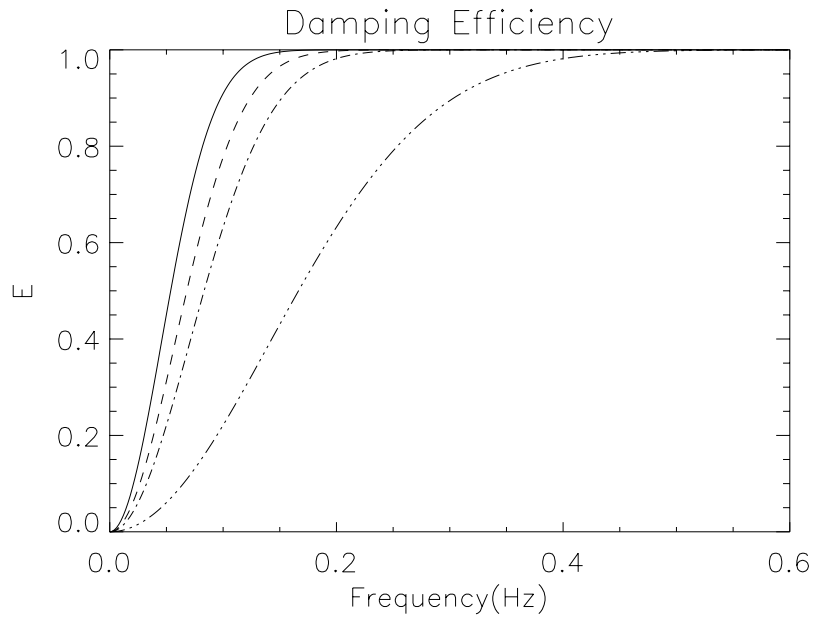

Fig. 3. Analytical estimates for the damping efficiency of the partially ionised region of the solar chromosphere as a function of frequency. The four lines represent the four magnetic field profile given by $\alpha=0.3$ (solid line). 0.35 (dash line), 0.4 (dot-dash line) and 0.6 (triple dot-dash line).

\section{Numerical method}

The propagation of Alfvén waves in a partially ionised plasma is modelled in 1D by numerically solving the non-ideal MHD equations, given here in dimensionless form.

$$
\begin{aligned}
\frac{\partial \rho}{\partial t}= & -\nabla \cdot(\rho \boldsymbol{v}) \\
\frac{\partial}{\partial t}(\rho \boldsymbol{v})= & -\nabla \cdot(\rho \boldsymbol{v} \boldsymbol{v})+(\boldsymbol{j} \wedge \boldsymbol{B})-\nabla P+\rho \boldsymbol{g} \\
\frac{\partial \boldsymbol{B}}{\partial t}= & \nabla \wedge(\boldsymbol{v} \wedge \boldsymbol{B})-\nabla \wedge\left(\eta \boldsymbol{j}_{\|}\right) \\
& -\nabla \wedge\left(\eta_{\mathrm{c}} \boldsymbol{j}_{\perp}\right) \\
\frac{\partial}{\partial t}(\rho \epsilon)= & -\nabla \cdot(\rho \boldsymbol{\epsilon} \boldsymbol{v})-P \nabla \cdot \boldsymbol{v} \\
& +\eta j_{\mathrm{par}}^{2}+\eta_{\mathrm{c}} j_{\text {perp }}^{2}
\end{aligned}
$$

which also include the Coulomb and Cowling resistivities $\eta=$ $1 / \sigma$ and $\eta_{\mathrm{c}}=1 / \sigma_{\mathrm{c}}$. $\boldsymbol{B}$ is the magnetic field, $\boldsymbol{j}$ is the current density, $\boldsymbol{v}$ is the velocity, $\mathrm{P}$ is the thermal pressure, and $\epsilon=$ $P /(\gamma-1) \rho$ is the specific energy density. The constants $\mu_{0}$ and $\gamma$ have the standard meanings of magnetic permittivity of free space and the ratio of specific heats respectively. Although for a partially ionised plasma $\gamma<5 / 3$ we found the value had little effect on the results so $5 / 3$ was used in all simulations.

It is worth noting that no viscous effects are included in these model equations as only collisional damping is being investigated in this work.

The simulations are carried out using a 2D MHD shock capturing code (Lare2d), applied in 1D only. It uses a staggered grid with density, pressure and specific energy density defined in the cell centres, magnetic field at the cell faces and velocities at the vertices. For further details see Arber et al. (2001). The code is adapted so that the resistive term in the induction equation updates the perpendicular and parallel components of current density separately, according to Eq. (16). Although the code is $1 \mathrm{D}$ all vector variables have 3 components.

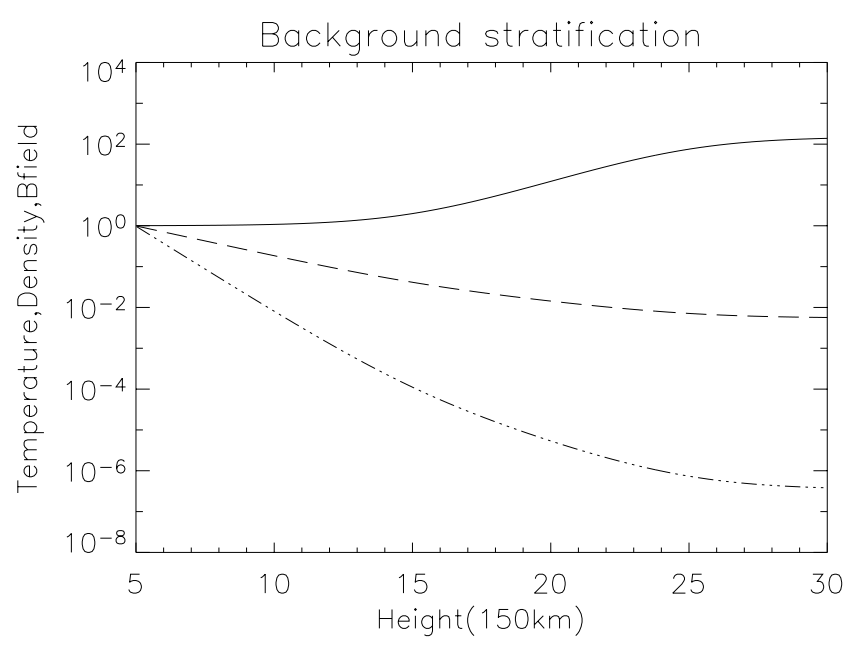

Fig. 4. The background plasma temperature (solid line), density (dotdash) and magnetic field (dash), where $\alpha=0.3$. The values are all given in dimensionless units.

The simulation domain extends $3000 \mathrm{~km}$ vertically, while the computational grid consists of 2000 cells. The number of cells vertically is restricted by the need to resolve the smallest wavelengths in the parametric study. The vertical boundary conditions are line-tied which are perfectly reflecting. Thus the simulation ends when the Alfvén waves have reached the upper boundary.

Using the VAL C model we construct the temperature profile, and the density is determined by solving the hydrostatic equilibrium equation.

$\frac{\partial P}{\partial z}=-\rho \boldsymbol{g}$

The background equilibrium is shown in Fig. 4 .

All quanities were non-dimensionalised by using values defined at $z=0$ from the VAL C model, thus

$$
\begin{aligned}
r^{*} & =150 \mathrm{~km} \\
\rho^{*} & =2.7 \times 10^{-4} \mathrm{~kg} / \mathrm{m}^{3} \\
v^{*} & =6515 \mathrm{~m} / \mathrm{s} \\
t^{*} & =23 \mathrm{~s} \\
T^{*} & =6420 \mathrm{~K} \\
P^{*} & =1.17 \times 10^{4} \mathrm{~Pa} \\
B^{*} & =1000 \mathrm{G} .
\end{aligned}
$$

Conversion to variables with dimensions merely requires the multiplication of internal variables by these values.

We model linear propagating Alfvén waves by driving the horizontal velocity $v_{x}$ continuously at the bottom of the domain with a sinusoidal driving function, the amplitude of which did not affect the results. Typical driving velocities were $600 \mathrm{~m} / \mathrm{s}$ and a range of 80 to $800 \mathrm{~m} / \mathrm{s}$ was tested.

As already mentioned in Sect. 2 the Cowling resistivity $\eta_{\mathrm{c}}$ is much larger than the Coulomb resistivity $\eta$ at chromospheric heights. In fact over the domain being simulated, the calculated value of $\eta$ using VALC values for the plasma never exceeds the value of numerical roundoff in the code, and hence all simulations are run with $\eta=0$. 

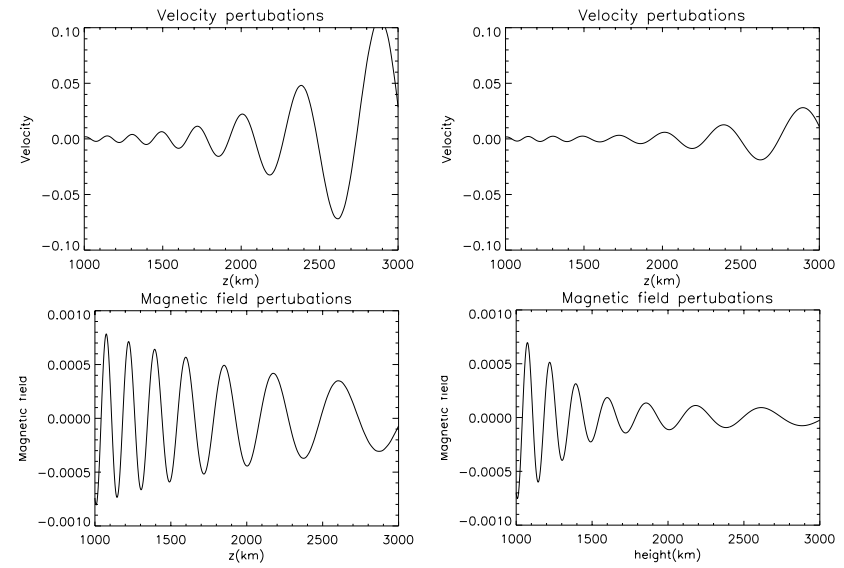

Fig. 5. Velocity (top slides) and magnetic field (bottom slides) perturbations against height for a typical run with $f=0.07 \mathrm{~Hz}$, The left hand slides are for the case of $\eta_{\mathrm{c}}=0$ and the right hand slides are for the $\eta_{\mathrm{c}}$ profile given by Fig. 2 . The velocity and magnetic field are given in dimensionless units.

The dissipation of these waves can then be investigated by observing the change in amplitudes of the perturbations. Figure 5 shows a typical profile of the perpendicular velocity and magnetic field perturbations with height for a driving frequency of $0.07 \mathrm{~Hz}$, for the case when no damping mechanism is applied and when collisonal friction damping is applied with the given profile of $\eta_{\mathrm{c}}$ (Fig. 2). As in Fig. 4 the value of $\alpha$ is 0.3 .

By comparing the propagation of Alfvén waves in the presence of a partially ionised region and without, we can study the efficiency of damping due to collsional friction of ions and neutrals in the partially ionised chromosphere.

As can be seen from Fig. 5, the gravitational stratification causes an increase in amplitude of the velocity pertubation and a decay in the corresponding magnetic field pertubations. This is a consequence of energy conservation in the travelling wave. For a rigorous mathematical treatment of this see Moortel \& Hood (2004). This makes a direct measuremnt of the efficiency of the collisional frictional damping non-trivial.

In order to obtain an estimate for the efficiency of collisional friction damping we compare the Poynting flux carried by the waves in the case when no damping mechanism is present and when there is damping due to ion-neutral collisions. The time-averaged Poynting flux for the Alfvén waves is given by

$\langle S\rangle=\frac{B_{0}\left\langle b_{x} v_{x}\right\rangle}{\mu_{0}}$

where $B_{0}$ is the vertical background magnetic field and $b_{x}, v_{x}$ are the perturbations in magnetic field and velocity. By calculating the ratio of Poynting flux at a height above the partially ionised region of the model chromosphere we can estimate the efficiency of the damping mechanism. The ratio of damped to undamped Poynting flux at a height of $2500 \mathrm{~km}$ above the surface (i.e. above the region of high Cowling conductivity) is given by

$\gamma=\frac{\langle S\rangle_{\text {damped }}}{\langle S\rangle_{\text {undamped }}}$

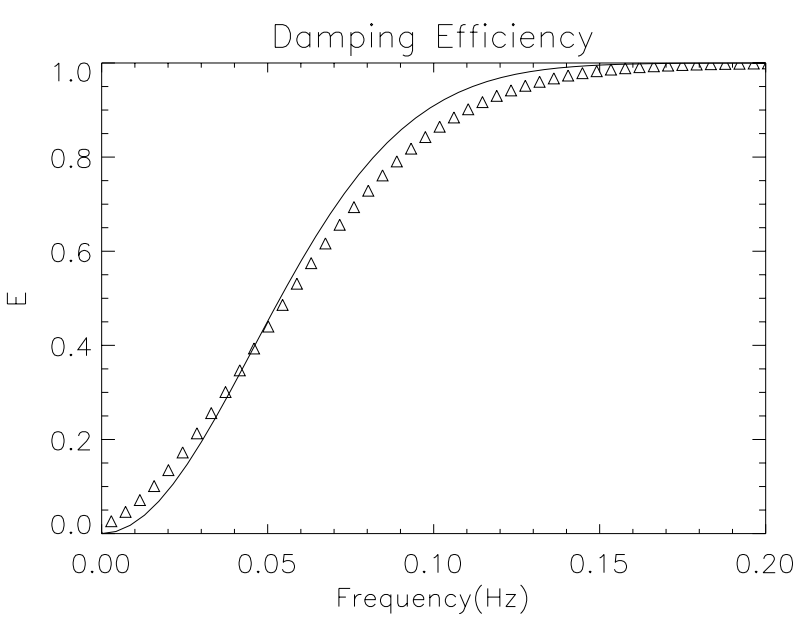

Fig. 6. Estimates of the efficiency of damping due to ion-neutral collisions for the magnetic field profile with power law $\alpha=0.3$. The solid line is the estimate due to analytic approches and the triangles represent the estimates obtained from numerical data.

and for direct comparison with analyic results the efficiency of damping is estimated simply by

$E_{\text {num }}=1-\gamma$.

Figures 6-9 show this estimate as a function of frequency, along with the estimate from the analytic approach (solid lines). The four different plots are for the four different magnetic field profiles, with $\alpha$ being $0.3,0.35,0.4$ and 0.6 respectively. As the power law for the magnetic field changes so does our estimate for the damping efficiency, as the value of $\eta_{\mathrm{c}}$ is dependant on $|\boldsymbol{B}|$.

The numerical data agrees best with the analytical estimates when $\alpha$ is smallest (see Fig. 6). For this profile we can see that the two approaches agree in the middle of the frequency range, but disagree at low and high frequencies. The differences at low frequencies are due to limitations in the procedure outined in Eqs. (38)-(40). The differences at high frequencies are due to the fact that the analytic approach is only applicable when the driving frequency is well below the critical value (Eq. (27)), i.e the linear damping approximation. The difference between numerical and analytic data increases as we increase the value of $\alpha$ (Figs. 6-9). By increasing this power in the magnetic field profile (Eq. (25)), we are decreasing the effect of ionneutral collisional friction damping, as $\eta_{\mathrm{c}}$ is now smaller. This increases the relative importance of any other effects on the wave amplitude. Thus the difference in numerical and analytic data is due to the fact that the numerical approach includes the stratification effects on the wave amplitudes, whereas the analytic approach does not.

From both analytical and numerical data we can see that Alfvén waves with frequencies below $0.01 \mathrm{~Hz}$ are unaffected by this damping mechanism, and propagate through the partially ionised region with little diffusion. Also waves with frequency above $0.6 \mathrm{~Hz}$ are completely damped by this mechanism. 


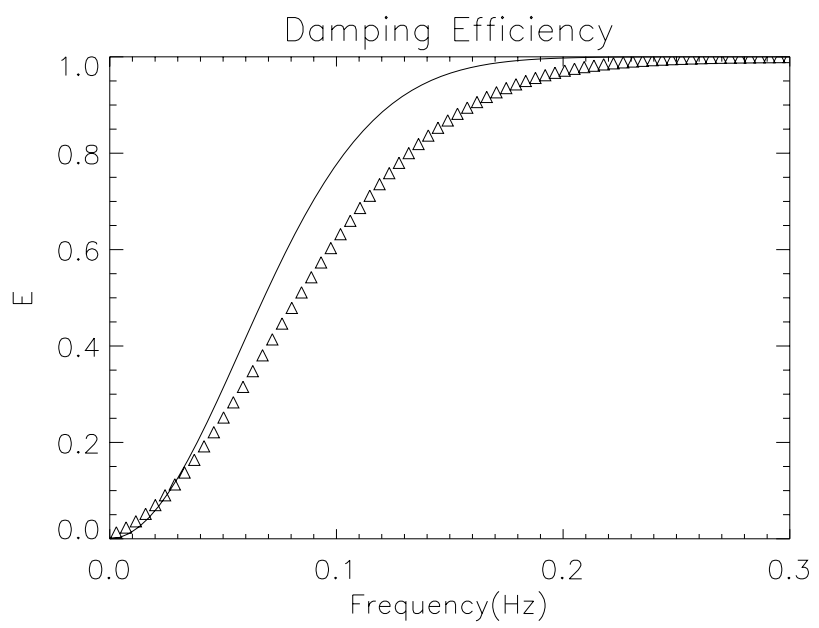

Fig. 7. Estimates of the efficiency of damping due to ion-neutral collisions for the magnetic field profile with power law $\alpha=0.35$. The solid line is the estimate due to analytic approches and the triangles represent the estimates obtained from numerical data.

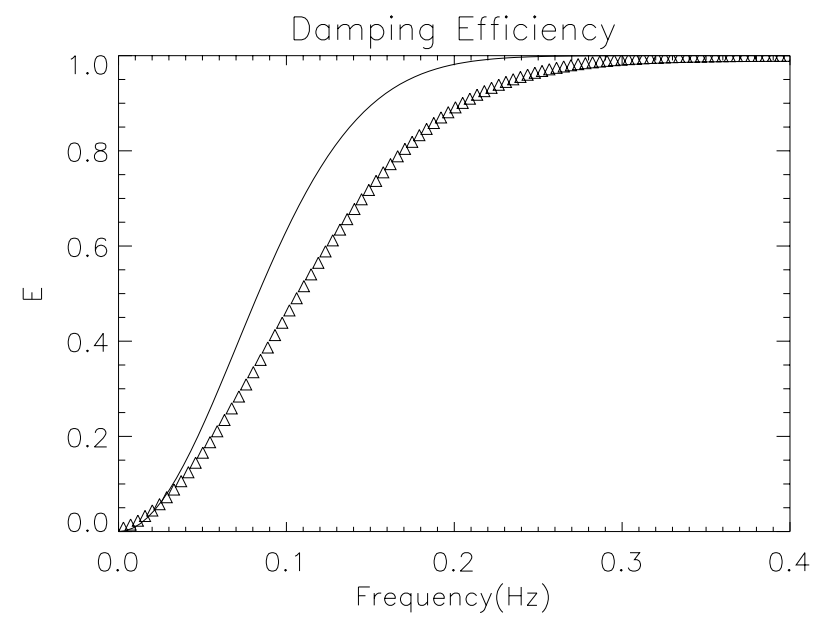

Fig. 8. Estimates of the efficiency of damping due to ion-neutral collisions for the magnetic field profile with power law $\alpha=0.4$. The solid line is the estimate due to analytic approches and the triangles represent the estimates obtained from numerical data.

\section{Conclusions}

We have estimated the efficiency of the partially ionised layer of the solar chromosphere in the damping of Alfvén waves generated at the surface. The estimates were based on analytic and numerical approaches, which agreed in the linear damping approximation. The damping mechanism was the collisional friction between neutral and ion species in a model Hydrogen plasma with temperature and density values taken from the VAL C model of the quiet Sun (Vernazza et al. 1981).

Alfvén waves of frequencies above $0.6 \mathrm{~Hz}$ were completely damped by the partially ionised layer in the chromosphere, whereas waves of frequency below $0.01 \mathrm{~Hz}$ were unaffected by the presence of neutrals and experienced no damping due to this mechanism. This lower result agrees with work conducted on MBP's and power spectra of horizontal motions at different heights in the atmosphere (Cranmer \& Ballegooijen 2005). They showed that using the WKB approximation the power

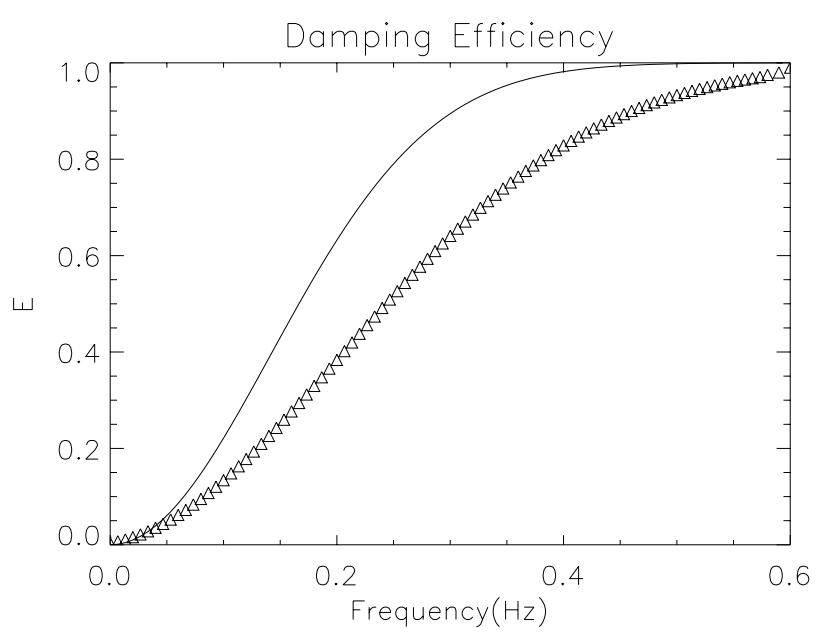

Fig. 9. Estimates of the efficiency of damping due to ion-neutral collisions for the magnetic field profile with power law $\alpha=0.6$. The solid line is the estimate due to analytic approches and the triangles represent the estimates obtained from numerical data.

spectra for motions with frequencies below $0.01 \mathrm{~Hz}$ are essentially unchanged as one progresses up the chromosphere to the transition region. This suggests that there is very little damping of low frequency waves due to any kind of dissipation mechanism in the solar chromosphere.

Although higher frequency waves are difficult to observe directly, photospheric motions can theoretically generate a large spectrum of Alfvén waves. The fact that the partially ionised layer completely damps any waves above $0.6 \mathrm{~Hz}$ for our magnetic field models means that high frequency waves in the upper atmosphere must have been created by other sources than photospheric motions.

This work is based on upward travelling waves generated at the photospheric level. Downward travelling waves from the corona would be reflected at the density contrast above the chromospheric region where the Cowling conductivity is large and it is unclear whether this damping mechanism would be important. The case of downward propagating waves will be subject to further investigation.

The numerical simulations were performed in the non-ideal MHD approximation with an additional term relating to the Cowling conductivity in the generalised Ohm's law (Eq. (16)). Previous work on Alfvén wave propagation in the lower solar atmosphere has used the WKB assumption, as used by (DePontieu 1999). This assumes that the change in wavelength, as well as variables, is small over a typical wavelength. They estimated that in this regime the ion-neutral collisions in the chromosphere damped waves of frequencies above $0.1 \mathrm{~Hz}$. Our results are in broad agrement with the results of De Pontieu et al. (2001) but as is clear from Figs. 6-9 the result is actually sensitive to the spreading out of the flux tube. Over the range of values of $\alpha$, chosen to match estimates of $|\boldsymbol{B}|$ from observations, the damping is effective over a range 0.1 to 0.6 depending on $\alpha$.

The model atmosphere was assumed to be magnetised, and thus the Generalised Ohm's law did not include the Hall term. In the upper photosphere, the electrons are tightly bound to the 
magnetic field whereas the ions are not. In this case there is a seperation electric field due to the neutrals drag on the nonmagnetised ion, which should be taken into account in the onefluid MHD equations. However, we showed that the damping of Alfvén waves is efficient at heights of $1000 \mathrm{~km}$ to $2000 \mathrm{~km}$ and in this region the plasma can be regarded as magnetised.

The form of the dissipation efficiency calculated from numerical data differed from that obtained from linear analytic appoximations. Small differences could be seen at low frequencies (due to errors in the estimation of small damping decrements). Differences also occured at higher frequencies, although both analytic and numerical estimates of the efficiency must converge to 1 at high frequencies. the difference at higher frequencies is due to the fact that the linear damping approximation is not valid (Eq. (27)).

Acknowledgements. This work was funded in part by the Particle Physics and Astronomy Research Council (PPA/S/S/2003/03728) and the Academic Research Collaboration Programme of the British Council in Austria. MK is grateful to the Austrian "Fonds zur Foerderungder wissenschaftlichen Forschung" (project P16919-N08) and 0eAD-RFBR grant (I.21/04)

\section{References}

Ambartsumyan, V. A. 1958, Theoretical Astrophysics (Pergamon Press)

Arber, T. D., Longbottom, A. W., Gerrard, C. L., \& Milne, A. M. 2001, JCP, 171,151

Braginskii, S. I. 1965, Transport processes in a Plasma, in Reviews of plasma phyics, Vol. 1 (New York: Consultants Bureau)
Brown, J. 1973, SoPh, 29, 421

Cowling, MagnetoHydrodynamics, Monographs on Astronomical Subjects (Adam Hilger), 1957

Cranmer, S. R., \& van Ballegooijen, A. A. 2005, ApJS, 156, 265

DeForest, C. E. 2004, ApJ, 617, L89

De Moortel, I., \& Hood, A. W. 2004, A\&A, 415,705

De Pontieu, B., \& Haerendel, G. 1998, A\&A, 338, 729

De Pontieu, B. 1999, A\&A, 347, 696

De Pontieu, B., Martens, P. C. H., \& Hudson, H. S. 2001, ApJ, 558, 859

Fossum, A., \& Carlsson, M. 2005, ApJ, 625, 556

Goodman, M. L. 2000, ApJ, 533, 501

Goodman, M. L. 2004, A\&A, 416, 1159

James, S. P., Erdélyi, R., De Pontieu, B. 2003, A\&A, 406, 715

Khodachenko, M. L., \& Zaitsev, V. V. 2001, Ap\&SS, 279, 389

Khodachenko, M. L., Arber, T. D., Rucker, H. O., \& Hanslmeier, A. 2004, A\&A, 422, 1073

Khodachenko, M. L., Arber, T. D., Rucker, H. O., Hanslmeier, Adv. Space Res., 2005, in press

Marsch, E., \& Tu, C.-Y. 1997, A\&A, 319 L17

Martinez-Pillet, V., Lites, B. W., \& Skumanich, A. 1997, ApJ, 474, 810

Nakariakov, V. M., Ofman, L., DeLuca, E., Roberts, B., \& Davila, J. M. 1999, Science, 285, 862

Piddington 1956, MNRAS, 116, 314

Pottasch, S. R., \& Thomas, R. N. 1959, ApJ, 130, 941

Spitzer, L. 1962, Physics of fully ionised gases (New York: Interscience)

Thomas \& Athay 1961, Physics of the Solar Chromosphere (3rd ed) (Oxford University Press)

Vernazza, J. E., Avrett, E. H., \& Loeser, R. 1981, ApJS, 45, 635 